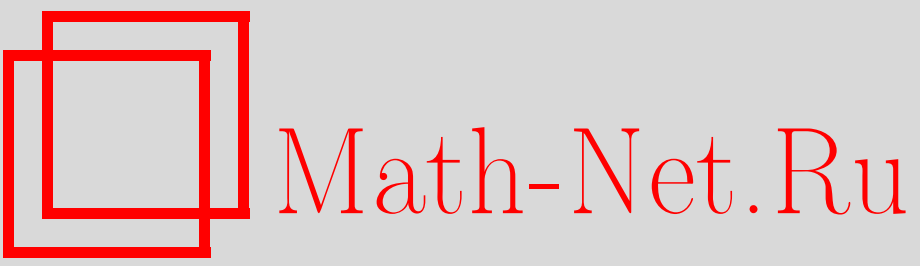

E. van Doorn, А. И. Зейфман, Т. Л. Панфилова, Оценки и асимптотика скорости сходимости для процессов рождения и гибели, Теория вероятн. и ее примен., 2009, том 54, выпуск 1, 18-38

DOI: https://doi.org/10.4213/tvp2497

Использование Общероссийского математического портала Math-Net.Ru подразумевает, что вы прочитали и согласны с пользовательским соглашением

http://www. mathnet.ru/rus/agreement

Параметры загрузки :

IP: 54.80 .97 .219

26 апреля 2023 г., 18:25:56 
ТЕОРИЯ ВЕРОЯТНОСТЕЙ

Tом 54

И ЕЕ ПРИМЕНЕНИЯ

Выпуск 1

2009

(c) 2009 г. ВАН ДООРН Э.А.*, ЗЕЙФМАН А. И.**,

ПАНФИЛОВА Т. Л.***

\title{
ОЦЕНКИ И АСИМПТОТИКА СКОРОСТИ СХОДИМОСТИ ДЛЯ ПРОЦЕССОВ РОЖДЕНИЯ И ГИБЕЛИ ${ }^{1)}$
}

\begin{abstract}
Первая часть статьи носит обзорный характер, в ней с той степенью подробности, которая позволяет применять описываемый подход, приводится основная база метода, разработанного одним из авторов в 1990-е годы для получения оценок и явных представлений для скоростей сходимости процессов рождения и гибели. Во второй части работы представлены новые результаты, полученные применением описанного метода к специальным классам процессов рождения и гибели, связанным с моделями среднего поля и системами обслуживания типа $M / M / N / N+R$, и относящиеся к асимптотике скорости сходимости в случае, когда число состояний процесса стремится к бесконечности.
\end{abstract}

Ключевые слова и фразы: процессы рождения и гибели, скорость сходимости, модель среднего поля.

1. Введение. Задачи, связанные с исследованием скорости сходимости к стационарному распределению для эргодических процессов рождения и гибели (далее ПРГ) в зависимости от интенсивностей процесса (интенсивностей рождения и гибели), изучались в литературе с использованием различных классов методов. Эти методы исследования имеют разную степень общности и, соответственно, в различной степени могут быть применены к решению указанных задач. Так, задача может быть сформулирована в терминах операторной теории, а соответствующие общие результаты можно попытаться применить к процессам рождения и гибели. Такой подход рассмотрен, например, в [4]. Можно использовать технику спектральных представлений переходных вероятностей

* Department of Applied Mathematics, University of Twente, P.O. Box 217, 7500 AE Enschede, The Netherlands; e-mail: e.a.vandoorn@utwente.nl

** Вологодский государственный педагогический университет, Институт проблем информатики РАН, ВНКЦ ЦЭМИ РАН, ул. С. Орлова, 6, 160035 Вологда, Россия; e-mail: zai@uni-vologda.ac.ru

*** Вологодский государственный педагогический университет, ул. С. Орлова, 6, 160035 Вологда, Россия.

1) Второй и третий авторы поддержаны РФФИ (грант № 06-01-00111) и государственным научным грантом Вологодской области. 
процессов рождения и гибели, развиваемую, начиная с работ С. Карлина и Дж. Мак-Грегора [16], [17], и сводящую рассматриваемые задачи к теории ортогональных полиномов. Этот подход применен, например, в [8]. В настоящей работе в качестве отправной точки выбирается система прямых уравнений Колмогорова для вероятностей состояний эргодической марковской цепи с непрерывным временем.

В статье преследуются две цели. Во-первых, это обзор и обсуждение метода исследования скорости сходимости для процессов рождения и гибели, инициированного заметкой [11], разработанного одним из авторов в начале 1990-х гг. (см. [29]-[31]) и развитого затем в совместных с Б.Л. Грановским работах (см. [12]-[14]). Во-вторых, представлен ряд новых результатов, полученных этим методом и связанных с асимптотикой скорости сходимости для некоторых специальных классов процессов рождения и гибели в случае, когда число состояний стремится к бесконечности. Метод основан на двух главных составляющих. Прежде всего, это понятие логарифмической нормь квадратной матрицы, введенное одновременно и независимо друг от друга С. М. Лозинским [20] и Г. Дальквистом [6] в качестве средства оценки погрешности численного интегрирования системы обыкновенных дифференциальных уравнений (см. также обзорные статьи [24] и [25]). Второй составляющей является специальное преобразование прямой системы Колмогорова. Будет рассмотрен случай конечной марковской цепи с непрерывным временем для обсуждения методологии без подробного описания технических деталей.

Статья построена следующим образом. Пункт 2 начинается с рассмотрения системы дифференциальных уравнений

$$
\mathbf{x}^{\prime}(t)=B \mathbf{x}(t), \quad t \geqslant 0,
$$

где квадратная матрица $B \equiv\left(b_{i j}\right)$ существенно положительна (т.е. все $b_{i j} \geqslant 0$ при $\left.i \neq j\right)$ и неразложима. Показано применение техники, основанной на использовании логарифмической нормы $B$, для представления и оценивания собственного значения $B$ с максимальной действительной частью и, соответственно, для представления и оценивания (подходящим образом определенной) нормы $\mathbf{x}(t)$ (теоремы $1-3)$. В п. 3 результаты п. 2 применяются для системы дифференциальных уравнений, получаемой специальным преобразованием прямой системы Колмогорова для вероятностей состояний конечной марковской цепи. В предположении существенной положительности матрицы системы этот подход позволяет получить представление и оценки скорости сходимости к стационарному распределению, а также соответствующие представление и оценки для (подходящим образом определенного) расстояния между распределением вероятностей состояний в момент $t \geqslant 0$ и стационарным распределением (теоремы 4 и 5). Далее, в п. 4 подходы и утверждения п. 3 применя- 
ются к процессам рождения и гибели, результаты для которых удается получить без всяких дополнительных условий (теоремы 7-9).

Выбирая конкретные классы интенсивностей рождения и гибели, мы можем теперь получать явные оценки скорости сходимости. В особых случаях удается даже указать точное значение параметра сходимости. Более общими являются ситуации, в которых удается получить асимптотические оценки (когда число состояний стремится к бесконечности) параметра сходимости. В пп. 5 и 6 изучаются два конкретных класса ПРГ, для которых получение такой асимптотики возможно. А именно, ПРГ, соответствующие моделям среднего поля, изучаются в п. 5, а процесс, описывающий число требований в системе обслуживания $M / M / N / N+R,-$ в п. 6 .

2. Предварительные утверждения. Будем обозначать векторстолбцы рассматриваемой длины, состоящие из нулей и единиц, соответственно $\mathbf{0}$ и $\mathbf{1}$. Неравенства для векторов и матриц понимаются покомпонентно, верхний индекс ${ }^{T}$ будет обозначать транспонирование. Матричную норму $\|\cdot\|$ будем предполагать индуцированной соответствующей векторной нормой, т.е. $\|A\|:=\max \{\|A \mathbf{a}\|:\|\mathbf{a}\|=1\}$. Тогда $\|A \mathbf{a}\| \leqslant\|A\|\|\mathbf{a}\|$ для любых матрицы $A$ и вектора а подходящих размерностей. Далее, для любой квадратной матрицы $A$ с собственным значением $\lambda$ имеем $|\lambda| \leqslant\|A\|$.

Если $B \equiv\left(b_{i j}\right)$ - матрица размера $n \times n$, а $\mathbf{x}(\cdot)$ определяется системой (1), то

$$
\mathbf{x}(t)=e^{t B} \mathbf{x}(0), \quad t \geqslant 0 .
$$

Хорошо известно (см., например, [25, лемма 1с] или $[13$, предложение 3$]$ ), что

$$
\left\|e^{t B}\right\| \leqslant e^{t \gamma(B)}, \quad t \geqslant 0
$$

и, следовательно,

$$
\|\mathbf{x}(t)\| \leqslant\left\|e^{t B}\right\|\|\mathbf{x}(0)\| \leqslant e^{t \gamma(B)}\|\mathbf{x}(0)\|, \quad t \geqslant 0
$$

где

$$
\gamma(B):=\lim _{h \downarrow 0} \frac{\|I+h B\|-1}{h}
$$

- логарифмическая норма матрицы $B$.

Обозначим собственные значения матрицы $B$ через $\lambda_{1}, \lambda_{2}, \ldots, \lambda_{n}$ и будем считать, что они расположены в порядке убывания их действительных частей, т.е. $\operatorname{Re} \lambda_{n} \leqslant \operatorname{Re} \lambda_{n-1} \leqslant \cdots \leqslant \operatorname{Re} \lambda_{1}$. Так как $e^{\lambda t}$ есть собственное значение матрицы $e^{t B}$, если $\lambda-$ собственное значение $B$, то

$$
e^{t \operatorname{Re} \lambda_{1}}=\left|e^{\lambda_{1} t}\right| \leqslant\left\|e^{t B}\right\|, \quad t \geqslant 0,
$$


и, следовательно, учитывая (2), получаем

$$
\operatorname{Re} \lambda_{1} \leqslant \gamma(B) \text {. }
$$

С другой стороны, используя формулу Гельфанда для спектрального радиуса (см., например, [23, теорема 10.13] или [27, теорема 3.4$]$ ), имеем

$$
\left\|e^{k B}\right\|^{1 / k} \rightarrow e^{\operatorname{Re} \lambda_{1}} \quad \text { при } \quad k \rightarrow \infty,
$$

а значит,

$$
\operatorname{Re} \lambda_{1}=\inf \left\{b \in \mathbf{R}:\left\|e^{t B}\right\|=O\left(e^{b t}\right) \text { при } t \rightarrow \infty\right\} \text {. }
$$

Учитывая, что $\left\|e^{t B}\right\|=\max \{\|\mathbf{x}(t)\|:\|\mathbf{x}(0)\|=1\}$, получаем также

$\operatorname{Re} \lambda_{1}=\inf \left\{b \in \mathbf{R}:\|\mathbf{x}(t)\|=O\left(e^{b t}\right)\right.$ при $t \rightarrow \infty$ для всех $\left.\mathbf{x}(0)\right\}$.

Далее будем предполагать, что $\|\cdot\|=\|\cdot\|_{1}$, т.е. что $\|\mathbf{a}\|=\sum_{i}\left|a_{i}\right|$ для любого вектора (столбца) $\mathbf{a} \equiv\left(a_{i}\right)$ и соответственно $\|A\|=\max _{j} \sum_{i}\left|a_{i j}\right|$ для любой матрицы $A \equiv\left(a_{i j}\right)$. Отсюда, в частности, получаем

$$
\gamma(B)=\max _{j}\left\{b_{j j}+\sum_{i \neq j}\left|b_{i j}\right|\right\} .
$$

Если матрица $B$ существенно положительна, то при достаточно большом $r \in \mathbf{R}$ матрица $B+r I$ неотрицательна, а тогда можно применить известные результаты Перрона-Фробениуса (см., например, [22] или [27]). Из них, в частности, вытекает, что $\lambda_{1}+r$, а следовательно, и $\lambda_{1}$ действительны. Полагая

$$
c_{\min }(B):=\min _{j} \sum_{i} b_{i j} \quad \text { и } \quad c_{\max }(B):=\max _{j} \sum_{i} b_{i j},
$$

имеем $\gamma(B)=c_{\max }(B)$, а с учетом $(5)$ и $(3)$ получим $\lambda_{1} \leqslant c_{\max }(B)$ и

$$
\|\mathbf{x}(t)\| \leqslant e^{t c_{\max }(B)}\|\mathbf{x}(0)\|, \quad t \geqslant 0,
$$

соответственно. Более того, в наших условиях оператор $e^{t B}$ положителен, т.е. $e^{t B} \mathbf{x} \geqslant \mathbf{0}$, если $\mathbf{x} \geqslant \mathbf{0}$ (см., например, [13, предложение 1] или $[27$, теорема 8.2]). В качестве следствия получаем отсюда

$$
\mathbf{x}(0) \geqslant \mathbf{0} \quad \Longrightarrow \quad\|\mathbf{x}(t)\| \geqslant e^{t c_{\min }(B)}\|\mathbf{x}(0)\|, \quad t \geqslant 0
$$

(см. $[13$, предложение 2$]$, причем условие $\sum_{i} b_{i j} \leqslant 0$ не является необходимым). Далее, используя (6), имеем оценку $\lambda_{1} \geqslant c_{\min }(B)$. Понятно, что в $(8)$ можно вместо $\mathbf{x}(0) \geqslant \mathbf{0}$ взять $\mathbf{x}(0) \leqslant \mathbf{0}$. В итоге получается следующее утверждение. 
Теорема 1. Пусть В существенно положительна, а $\mathbf{x}(0)-$ произвольный вектор начальных условий. Тогда

$$
c_{\min }(B) \leqslant \lambda_{1} \leqslant c_{\max }(B)
$$

$u$

$$
e^{t c_{\min }(B)}\|\mathbf{x}(0)\| \leqslant\|\mathbf{x}(t)\| \leqslant e^{t c_{\max }(B)}\|\mathbf{x}(0)\|, \quad t \geqslant 0,
$$

причем левое неравенство в (10) справедливо при выполнении одного из дополнительных условий $\mathbf{x}(0) \geqslant \mathbf{0}$ или $\mathbf{x}(0) \leqslant \mathbf{0}$.

Система (1) не изменится, если мы заменим $\mathbf{x}(0)$ на $-\mathbf{x}(0)$, а $B$ на $-B$, так что можно попытаться применить те же рассуждения и для $-B$. Однако, поскольку $-B$ не является существенно положительной, когда $B$ существенно положительна, можно использовать только рассуждения, не опирающиеся на существенную положительность. Положим

$$
s_{\min }(B):=\min _{j}\left\{b_{j j}-\sum_{i \neq j} b_{i j}\right\} \quad \text { и } \quad s_{\max }(B):=\max _{j}\left\{b_{j j}-\sum_{i \neq j} b_{i j}\right\} .
$$

Замечая, что $-\gamma(-B)=\min _{j}\left\{b_{j j}-\sum_{i \neq j}\left|b_{i j}\right|\right\}=s_{\min }(B)$, если $B$ существенно положительна, получаем следующее утверждение.

Теорема 2. Пусть В существенно положительна, а $\mathbf{x}(0)$ произвольно. Тогда

$$
s_{\min }(B) \leqslant \operatorname{Re} \lambda_{n}
$$

$u$

$$
e^{t s_{\min }(B)}\|\mathbf{x}(0)\| \leqslant\|\mathbf{x}(t)\|, \quad t \geqslant 0 .
$$

Очевидно, что $s_{\min }(B) \leqslant c_{\min }(B)$, если $B$ существенно положительна, так что оценка (13) становится содержательной только в случае, когда вектор $\mathbf{x}(0)$ имеет координаты разных знаков.

Используя предположение о существенной положительности $B$, можно несколько улучшить результаты теоремы 1 . Отметим вначале, что для произвольной невырожденной матрицы $T$ имеем

$$
T \mathbf{x}^{\prime}(t)=\left(T B T^{-1}\right) T \mathbf{x}(t), \quad t \geqslant 0,
$$

и, применяя теорему 1 не к $B$, а к $T B T^{-1}$, получим

$$
e^{t c_{\min }\left(T B T^{-1}\right)}\|\mathbf{x}(0)\|_{T} \leqslant\|\mathbf{x}(t)\|_{T} \leqslant e^{t c_{\max }\left(T B T^{-1}\right)}\|\mathbf{x}(0)\|_{T}, \quad t \geqslant 0
$$

где для $(n \times n)$-матрицы $T$ векторная норма $\|\cdot\|_{T}$ определяется по формуле

$$
\|\mathbf{a}\|_{T}:=\|T \mathbf{a}\|,
$$

а для выполнения левого неравенства в (14) достаточно, чтобы выполнялось неравенство $T \mathbf{x}(0) \geqslant \mathbf{0}$ или $T \mathbf{x}(0) \leqslant \mathbf{0}$. 
Далее, выберем снова $r \in \mathbf{R}$ настолько большим, чтобы матрица $B+r I$ стала неотрицательной. Тогда в соответствии с теоремой Коллатца-Виланда (см., например, [22, с. 666-669] или [27, теорема 2.9]) имеем для любой диагональной матрицы $D$ с положительными диагональными элементами следующие возможности для спектрального радиуса $\rho(B+r I)$ матрицы $B+r I$ :

$$
c_{\min }\left(D(B+r I) D^{-1}\right)<\rho(B+r I)<c_{\max }\left(D(B+r I) D^{-1}\right)
$$

или

$$
c_{\min }\left(D(B+r I) D^{-1}\right)=\rho(B+r I)=c_{\max }\left(D(B+r I) D^{-1}\right) .
$$

Более того, равенство имеет место тогда и только тогда, когда $D \mathbf{1}$ есть положительный собственный вектор матрицы $B+r I$, соответствующий собственному значению $\rho(B+r I)$ (единственный с точностью до скалярного множителя). Замечая, что $\rho(B+r I)=\lambda_{1}+r$, и заменяя $T$ на $D$ в (14), получаем следующее обобщение теоремы 1.

Теорема 3. Пусть В существенно положительна, а $\mathbf{x}(0)-$ произвольный ненулевой вектор. Тогда для любой диагональной матриць $D$ с положительными элементами справедливы неравенства

$$
c_{\min }\left(D B D^{-1}\right) \leqslant \lambda_{1} \leqslant c_{\max }\left(D B D^{-1}\right)
$$

$u$

$$
e^{t c_{\min }\left(D B D^{-1}\right)}\|\mathbf{x}(0)\|_{D} \leqslant\|\mathbf{x}(t)\|_{D} \leqslant e^{t c_{\max }\left(D B D^{-1}\right)}\|\mathbf{x}(0)\|_{D}, \quad t \geqslant 0
$$

где левое неравенство в (16) заведомо выполняется, если $\mathbf{x}(0) \geqslant \mathbf{0}$ или $\mathbf{x}(0) \leqslant \mathbf{0}$. Граничные значения в (15) (u, следовательно, в (16), если $\mathbf{x}(0) \geqslant \mathbf{0}$ или $\mathbf{x}(0) \leqslant \mathbf{0})$ достигаются тогда и только тогда, когда D1 является положительным собственныл вектором матрицы $B$, соответствуюшим собственному значению $\lambda_{1}$.

Отсюда немедленно вытекает такое следствие (см. также [27, задача 8.2.6]).

Следствие 1. Если $B$ существенно положительна, а $D-\partial и а$ гональная матрича, то для любого ненулевого начального условия $\mathbf{x}(0) \geqslant \mathbf{0}$ равенство

$$
\|\mathbf{x}(t)\|_{D}=e^{\lambda_{1} t}\|\mathbf{x}(0)\|_{D}, \quad t \geqslant 0
$$

выполняется тогда и только тогда, когда D1 - положительный собственный вектор матриџь $B$, соответствующий собственному значению $\lambda_{1}$. 
Для полноты изложения и удобства дальнейших ссылок отметим также результат, получающийся при замене $B$ на $D B D^{-1}$ и $\mathbf{x}(t)$ на $D \mathbf{x}(t)$ в (1) и (13), а именно, неравенство

$$
e^{t s_{\min }\left(D B D^{-1}\right)}\|\mathbf{x}(0)\|_{D} \leqslant\|\mathbf{x}(t)\|_{D}, \quad t \geqslant 0,
$$

справедливое для любого вектора $\mathbf{x}(0)$ и любой диагональной матрицы $D$ с положительными диагональными элементами. С учетом теоремы 3 , очевидно, что это неравенство становится содержательным только в случае, когда вектор $\mathbf{x}(0)$ имеет координаты разных знаков, поскольку $s_{\min }\left(D B D^{-1}\right) \leqslant c_{\min }\left(D B D^{-1}\right)$.

3 а м е ч а н и е. Некоторое небольшое обобщение теоремы 3 и неравенства (18) можно получить, рассматривая вместо (1) систему

$$
\mathbf{y}^{\prime}(t)=q(t) B \mathbf{y}(t), \quad t \geqslant 0,
$$

где (скалярная) функция $q(\cdot)$ предполагается неотрицательной и локально интегрируемой на $[0, \infty)$. В этом случае получаем

$$
\mathbf{y}(t)=e^{\tilde{q}(t) B} \mathbf{y}(0), \quad t \geqslant 0,
$$

где $\widetilde{q}(t):=\int_{0}^{t} q(u) d u$. Полагая $\mathbf{x}(t):=\mathbf{y}\left(\widetilde{q}^{-1}(t)\right)$, можно свести систему (19) к системе (1), применить (16)-(18) и сформулировать результаты в терминах $\mathbf{y}(\cdot)$. Это можно проделать, заменяя $\mathbf{x}(\cdot)$ на $\mathbf{y}(\cdot)$ в (16)-(18) и записывая $\widetilde{q}(t)$ вместо $t$ в показателе.

Если же $B$ не является существенно положительной, можно попытаться найти преобразование подобия $C=T B T^{-1}$ такое, чтобы $C$ стала существенно положительной, а затем применить теорему 3 к $C$ вместо $B$ для получения оценок $\lambda_{1}$.

3. Сходимость марковских цепей. Рассмотрим марковскую цепь с непрерывным временем $\mathscr{X} \equiv\{X(t), t \geqslant 0\}$, принимающую значения из множества $\{0,1, \ldots, n\}$ с $q$-матрицей $Q \equiv\left(q_{i j}\right)$. Вероятности состояний цепи $p_{j}(t):=\operatorname{Pr}\{X(t)=j\}, j=0,1, \ldots, n$, определяются начальным распределением и системой прямых уравнений Колмогорова

$$
\mathbf{p}^{\prime}(t)=Q^{T} \mathbf{p}(t), \quad t \geqslant 0,
$$

где $\mathbf{p}(t) \equiv\left(p_{0}(t), p_{1}(t), \ldots, p_{n}(t)\right)^{T}$. Будем предполагать, что цепь $\mathscr{X}$ консервативна и неразложима, а значит, имеет единственное стационарное распределение, определяемое вектором $\boldsymbol{\pi} \equiv\left(\pi_{0}, \pi_{1}, \ldots, \pi_{n}\right)^{T}$. Распределение $\boldsymbol{\pi}$ удовлетворяет условию $Q^{T} \boldsymbol{\pi}=\mathbf{0}$, а $\mathbf{p}(t)$ сходится к $\boldsymbol{\pi}$ при $t \rightarrow \infty$ при любом начальном распределении $\mathbf{p}(0)$. Нас будет интересовать параметр сходимости (decay parameter) $\beta$, определяемый формулой

$$
\beta:=\sup \left\{a>0:\|\mathbf{p}(t)-\boldsymbol{\pi}\|=O\left(e^{-a t}\right) \text { при } t \rightarrow \infty \text { для всех } \mathbf{p}(0)\right\} .
$$


Поскольку $\sum_{k=0}^{n} p_{k}(t)=\sum_{k=0}^{n} \pi_{k}=1$, имеем очевидное неравенство

$$
0 \leqslant\left|p_{0}(t)-\pi_{0}\right| \leqslant \sum_{k=1}^{n}\left|p_{k}(t)-\pi_{k}\right|, \quad t \geqslant 0 .
$$

Теперь, полагая $\mathbf{x}(t):=\left(p_{1}(t)-\pi_{1}, p_{2}(t)-\pi_{2}, \ldots, p_{n}(t)-\pi_{n}\right)^{T}$, приходим к оценке

$$
\|\mathbf{x}(t)\| \leqslant\|\mathbf{p}(t)-\boldsymbol{\pi}\| \leqslant 2\|\mathbf{x}(t)\|, \quad t \geqslant 0
$$

а значит,

$$
\beta=\sup \left\{a>0:\|\mathbf{x}(t)\|=O\left(e^{-a t}\right) \text { при } t \rightarrow \infty \text { для всех } \mathbf{x}(0)\right\} .
$$

Отметим также, что из системы (20) вытекает

$$
\begin{aligned}
p_{i}^{\prime}(t) & =q_{0 i}\left(p_{0}(t)-\pi_{0}\right)+\sum_{j=1}^{n} q_{j i}\left(p_{j}(t)-\pi_{j}\right) \\
& =\sum_{j=1}^{n}\left(q_{j i}-q_{0 i}\right)\left(p_{j}(t)-\pi_{j}\right), \quad i=1,2, \ldots, n, \quad t \geqslant 0,
\end{aligned}
$$

так что

$$
x_{i}^{\prime}(t)=\sum_{j=1}^{n}\left(q_{j i}-q_{0 i}\right) x_{j}(t), \quad i=1,2, \ldots, n, \quad t \geqslant 0 .
$$

Таким образом, $\mathbf{x}(\cdot)$ удовлетворяет системе дифференциальных уравнений (1), а значит, $\mathbf{x}(t)=e^{t B} \mathbf{x}(0)$, где матрица $B \equiv\left(b_{i j}\right)$ определяется следующим образом:

$$
b_{i j}:=q_{j i}-q_{0 i}, \quad i, j=1,2, \ldots, n .
$$

Как и в предыдущем пункте, обозначим собственные значения матрицы $B$ через $\lambda_{1}, \lambda_{2}, \ldots, \lambda_{n}$ и будем считать, что $\operatorname{Re} \lambda_{n} \leqslant \operatorname{Re} \lambda_{n-1} \leqslant$ $\cdots \leqslant \operatorname{Re} \lambda_{1}$. Поскольку марковская цепь $\mathscr{X}$ консервативна и неразложима, нулевое собственное значение матрицы $Q$ является простым, а все остальные имеют отрицательные действительные части. Следовательно, $\lambda_{1}, \lambda_{2}, \ldots, \lambda_{n}$ - ненулевые собственные значения $Q$, причем

$$
\operatorname{Re} \lambda_{n} \leqslant \operatorname{Re} \lambda_{n-1} \leqslant \cdots \leqslant \operatorname{Re} \lambda_{1}<0 .
$$

В результате, используя (6) и (23), получаем

$$
\beta=-\operatorname{Re} \lambda_{1},
$$

а значит, изучение скорости сходимости марковской цепи $\mathscr{X}$ с $q$ матрицей $Q \equiv\left(q_{i j}\right)$ сводится в значительной степени к исследованию собственного значения с наибольшей действительной частью для матрицы $B \equiv\left(b_{i j}\right)$, определяемой формулой $(24)$. 
Хотя исходная матрица $Q$ существенно положительна, матрица $B$, вообще говоря, таковой не является. А тогда, как упоминалось в конце предыдущего пункта, прежде чем применять теорему 3 , надо подобрать преобразование $C=T B T^{-1}$ (если оно, конечно, есть) такое, что $C$ уже является существенно положительной. В следующем пункте мы рассмотрим класс таких преобразований для ПРГ, а пока будем просто предполагать существование. В качестве следствия такого предположения мгновенно получаем действительность $\lambda_{1}$. Очевидно, далее, что если все-таки сама матрица $B$ оказывается существенно положительной, то можно взять $T=I$. Можно еще добавить, что в соответствии с $[13$, теорема 2] подходящее преобразование существует всегда в случае, если цепь $\mathscr{X}$ является обратимой (reversible) (в этом случае все собственные значения действительны).

В предположении существования невырожденного преобразования $T$ такого, что $C=T B T^{-1}$ существенно положительна, можно применить первую часть теоремы 3 к $C$ и, с учетом (25), получить следующую информацию о $\beta$, а значит, и о скорости сходимости марковской цепи $\mathscr{X}$.

Теорема 4. Пусть $T$ - невырожденная матрица такая, что $C=$ $T B T^{-1}$ существенно положительна, и пусть $D-$ диагональная матрица с положительныли диагональными элементами. Тогда

$$
c_{\min }\left(D T(-B)(D T)^{-1}\right) \leqslant \beta \leqslant c_{\max }\left(D T(-B)(D T)^{-1}\right),
$$

причем равенство достигается тогда и только тогда, когда D1 является собственным вектором матричь $C$, соответствуюиим собственному значению $\lambda_{1}=-\beta$.

Полагая $\mathbf{z}(\cdot):=T \mathbf{x}(\cdot)$, имеем

$$
\mathbf{z}^{\prime}(t)=T B T^{-1} \mathbf{z}(t)=C \mathbf{z}(t), \quad t \geqslant 0,
$$

причем $\mathbf{x}(\cdot)$ удовлетворяет системе дифференциальных уравнений (1). Отметим, далее, что

$$
\|\mathbf{z}(t)\|_{D}=\|D T \mathbf{x}(t)\| \leqslant\|D T\|\|\mathbf{x}(t)\|, \quad t \geqslant 0
$$

и

$$
\|\mathbf{x}(t)\|=\left\|(D T)^{-1} D \mathbf{z}(t)\right\| \leqslant\left\|(D T)^{-1}\right\|\|\mathbf{z}(t)\|_{D}, \quad t \geqslant 0,
$$

причем с учетом $(22)$

$$
\|D T\|^{-1}\|\mathbf{z}(t)\|_{D} \leqslant\|\mathbf{p}(t)-\boldsymbol{\pi}\| \leqslant 2\left\|(D T)^{-1}\right\|\|\mathbf{z}(t)\|_{D}, \quad t \geqslant 0 .
$$

Теперь можно применить к $\mathbf{z}(\cdot)$ вторую часть теоремы 3 и оценку (18). Используя неравенства (27), можно легко получить следующие явные оценки для $\|\mathbf{p}(t)-\boldsymbol{\pi}\|$. 
Теорема 5. Пусть невьрожденная матрица $T$ такова, что $C=$ $T B T^{-1}$ существенно положительна, и пусть $D$ - диагональная матрица с положительными диагональными элементами. Тогда для любого начального распределения $\mathbf{p}(0)$ вылолнень неравенства

$$
\begin{array}{ll}
\|\mathbf{p}(t)-\boldsymbol{\pi}\| \leqslant \kappa e^{-c_{\min } t}\|\mathbf{p}(0)-\boldsymbol{\pi}\|, & t \geqslant 0, \\
\|\mathbf{p}(t)-\boldsymbol{\pi}\| \geqslant \frac{1}{\kappa} e^{-s_{\max } t}\|\mathbf{p}(0)-\boldsymbol{\pi}\|, & t \geqslant 0,
\end{array}
$$

где $\kappa:=2\|D T\|\left\|(D T)^{-1}\right\|, c_{\min } u c_{\max }$ обозначают соответственно левую и правую части $(26)$, а $s_{\max }:=s_{\max }\left(D T(-B)(D T)^{-1}\right)$. Кроме того, если все $p_{i}(0) \geqslant \pi_{i}, i=1,2, \ldots, n$, или все $p_{i}(0) \leqslant \pi_{i}$ при $i=1,2, \ldots, n$, mo

$$
\|\mathbf{p}(t)-\boldsymbol{\pi}\| \geqslant \frac{1}{\kappa} e^{-c_{\max } t}\|\mathbf{p}(0)-\boldsymbol{\pi}\|, \quad t \geqslant 0
$$

Отметим, что условие (30), в частности, выполнено, если начальное распределение вероятностей сконцентрировано в нуле. Разумеется, оценка (29) становится неэффективной, если (30) выполнено, поскольку $s_{\max }<c_{\max }$.

Поскольку $B$ и $D T B(D T)^{-1}$ имеют одинаковый спектр, теорема 2 гарантирует, что $-s_{\max } \leqslant \operatorname{Re} \lambda_{n}$. В следующем пункте мы получим более точные оценки для $\lambda_{n}-$ собственного значения матрицы $Q$ с минимальной действительной частью в случае ПРГ.

Отметим наконец, что справедливо следующее утверждение.

Теорема 6. Если для некоторой невырожденной матриць $T$ матрица $C=T B T^{-1}$ является существенно положительной, то существует неотрииательная матрииа $\Delta$ такая, что для любого начального распределения $\mathbf{p}(0)$, удовлетворяющего условию $p_{i}(0) \geqslant \pi_{i}$ при $i=1,2, \ldots, n\left(\right.$ или $p_{i}(0) \leqslant \pi_{i}$ при $\left.i=1,2, \ldots, n\right)$,

$$
\|\mathbf{p}(t)-\boldsymbol{\pi}\|_{\Delta}=e^{-\beta t}\|\mathbf{p}(0)-\boldsymbol{\pi}\|_{\Delta}, \quad t \geqslant 0
$$

Д о к а з а т е л ь с т в о. Выберем диагональную матрицу $D$ так, чтобы $D \mathbf{1}$ был положительным собственным вектором $C$, соотвествующим $\lambda_{1}$. Тогда из следствия 1, примененного к $C$ и $\mathbf{z}(\cdot)$, получаем

$$
\|\mathbf{z}(t)\|_{D}=e^{-\beta t}\|\mathbf{z}(0)\|_{D}, \quad t \geqslant 0
$$

Если $\Delta$ - матрица с нулевыми первой строкой и первым столбцом и с матрицей $D T$ в качестве остальной части, то получаем

$$
\|\mathbf{p}(t)-\boldsymbol{\pi}\|_{\Delta}=\|\mathbf{x}(t)\|_{D T}=\|\mathbf{z}(t)\|_{D}, \quad t \geqslant 0
$$

откуда и следует требуемое утверждение. 
В следующем пункте будет показано, как общая техника, описанная здесь для общего анализа скорости сходимости конечной марковской цепи с непрерывным временем, работает и позволяет получить явные представления и оценки в более специальном случае процессов рождения и гибели.

4. Процессы рождения и гибели. В этом пункте предполагается, что рассматриваемая марковская цепь $\mathscr{X}$ является процессом рождения и гибели (ПРГ), т.е. ее $q$-матрица имеет вид

$$
Q=\left(\begin{array}{cccccc}
-a_{0} & a_{0} & 0 & \cdots & 0 & 0 \\
b_{1} & -\left(a_{1}+b_{1}\right) & a_{1} & \cdots & 0 & 0 \\
\vdots & \vdots & \vdots & \ddots & \vdots & \vdots \\
0 & 0 & 0 & \cdots & -\left(a_{n-1}+b_{n-1}\right) & a_{n-1} \\
0 & 0 & 0 & \cdots & b_{n} & -b_{n}
\end{array}\right)
$$

с положительными интенсивностями рождения $a_{i}, 0 \leqslant i<n$, и интенсивностями гибели $b_{i}, 0<i \leqslant n$. Как известно, ненулевые собственные значения $\lambda_{1}, \lambda_{2}, \ldots, \lambda_{n}$ матрицы $Q$ действительны, различны и отрицательны (см., например, [17]). В предыдущем пункте было отмечено, что все эти собственные значения являются и точками спектра матрицы $B$ из (24), которая теперь выглядит следующим образом:

$$
B=\left(\begin{array}{cccccc}
-\left(a_{0}+a_{1}+b_{1}\right) & b_{2}-a_{0} & -a_{0} & \cdots & -a_{0} & -a_{0} \\
a_{1} & -\left(a_{2}+b_{2}\right) & b_{3} & \cdots & 0 & 0 \\
\vdots & \vdots & \vdots & \ddots & \vdots & \vdots \\
0 & 0 & 0 & \cdots & -\left(a_{n-1}+b_{n-1}\right) & b_{n} \\
0 & 0 & 0 & \cdots & a_{n-1} & -b_{n}
\end{array}\right) .
$$

Выбирая в качестве $T$ верхнетреугольную матрицу с единицами на диагонали и над ней, получаем

$$
T=\left(\begin{array}{cccccc}
1 & 1 & 1 & \cdots & 1 & 1 \\
0 & 1 & 1 & \cdots & 1 & 1 \\
\vdots & \vdots & \vdots & \ddots & \vdots & \vdots \\
0 & 0 & 0 & \cdots & 1 & 1 \\
0 & 0 & 0 & \cdots & 0 & 1
\end{array}\right), \quad T^{-1}=\left(\begin{array}{cccccc}
1 & -1 & 0 & \cdots & 0 & 0 \\
0 & 1 & -1 & \cdots & 0 & 0 \\
\vdots & \vdots & \vdots & \ddots & \vdots & \vdots \\
0 & 0 & 0 & \cdots & 1 & -1 \\
0 & 0 & 0 & \cdots & 0 & 1
\end{array}\right)
$$

а преобразование подобия $C=T B T^{-1}$ приводит нас к существенно положительной матрице 


$$
C=\left(\begin{array}{cccccc}
-\left(a_{0}+b_{1}\right) & b_{1} & 0 & \cdots & 0 & 0 \\
a_{1} & -\left(a_{1}+b_{2}\right) & b_{2} & \cdots & 0 & 0 \\
\vdots & \vdots & \vdots & \ddots & \vdots & \vdots \\
0 & 0 & 0 & \cdots & -\left(a_{n-2}+b_{n-1}\right) & b_{n-1} \\
0 & 0 & 0 & \cdots & a_{n-1} & -\left(a_{n-1}+b_{n}\right)
\end{array}\right) .
$$

Теперь можно применить теорему 4 и после небольших вычислений получить следующее утверждение, которое в полной общности было впервые получено в [31].

Теорема 7. Параметр сходимости $\beta$ для ПРГ с q-матричей (31) удовлетворяет условию

$$
\max _{\mathbf{d}>\mathbf{0}}\left\{\min _{1 \leqslant i \leqslant n} \alpha_{i}\right\}=\beta=\min _{\mathbf{d}>\mathbf{0}}\left\{\max _{1 \leqslant i \leqslant n} \alpha_{i}\right\},
$$

где $\mathbf{d} \equiv\left(d_{1}, d_{2}, \ldots, d_{n}\right) u$

$$
\alpha_{i}:=a_{i-1}+b_{i}-\frac{d_{i+1}}{d_{i}} a_{i}-\frac{d_{i-1}}{d_{i}} b_{i-1}, \quad i=1,2, \ldots, n,
$$

причем $a_{n}=b_{0}=d_{0}=d_{n+1}=0$.

Имеется обширная литература, связанная с результатами, близкими к теореме 7 , основанная на различных подходах, а в ряде работ исследован и случай эргодического ПРГ со счетным пространством состояний. Укажем здесь работы [2]-[4], [7]-[9], [12], [13], [15], [18], [29]-[32], см. также приведенные в этих работах ссылки.

Информацию о $\lambda_{n}$ - наименьшем собственном значении матрицы $Q$, а значит, и матрицы $C$, можно получить, заметив, что $-\lambda_{n}$ является наибольшим собственным значением матрицы $-C$. Поскольку характеристический полином $P_{r}(\lambda)$ для главной подматрицы порядка $r$ матрицы $-C$ удовлетворяет рекуррентным формулам

$$
\begin{aligned}
& P_{r}(\lambda)=\left(a_{r-1}+b_{r}-\lambda\right) P_{r-1}(\lambda)-a_{r-1} b_{r-1} P_{r-2}(\lambda), \quad r=2,3, \ldots, n, \\
& P_{0}(\lambda)=1, \quad P_{1}(\lambda)=a_{0}+b_{1}-\lambda,
\end{aligned}
$$

понятно, что $P_{n}(\lambda)$, а следовательно, и спектр матрицы $-C$ не меняется при изменении знаков ее внедиагональных элементов. Другими словами, $-\lambda_{1}=\beta<-\lambda_{2}<\cdots<-\lambda_{n}$ являются собственными значениями неотрицательной (и неразложимой, а значит, существенно положительной) матрицы $C^{*}$, которая получается из $C$ изменением знаков ее диагональных элементов. Применяя теперь теорему 3 к $C^{*}$, получаем следующее утверждение.

Теорема 8. Пусть $\chi=-\lambda_{n}$, где $\lambda_{n}$ - наименьшее собственное значение q-матричь (31). Тогда для ұ справедливь представления

$$
\max _{\mathbf{d}>\mathbf{0}}\left\{\min _{1 \leqslant i \leqslant n} \zeta_{i}\right\}=\chi=\min _{\mathbf{d}>\mathbf{0}}\left\{\max _{1 \leqslant i \leqslant n} \zeta_{i}\right\},
$$


где $\mathbf{d} \equiv\left(d_{1}, d_{2}, \ldots, d_{n}\right) u$

$$
\zeta_{i}:=a_{i-1}+b_{i}+\frac{d_{i+1}}{d_{i}} a_{i}+\frac{d_{i-1}}{d_{i}} b_{i-1}, \quad i=1,2, \ldots, n,
$$

причем $a_{n}=b_{0}=d_{0}=d_{n+1}=0$.

Приведем теперь соответствующие экспоненциальные оценки для $\|\mathbf{p}(t)-\boldsymbol{\pi}\|$. Результаты в основном были получены в [29] и [31].

Теорема 9. Пусть $\mathscr{X}-$ ПРГ с q-матричей (31). Тогда для любого начального распределения $\mathbf{p}(0)$ и любого вектора $\mathbf{d} \equiv$ $\left(d_{1}, d_{2}, \ldots, d_{n}\right)$ такого, ито $d:=\min _{i}\left\{d_{i}\right\}>0$, справедливь оченки

$$
\begin{aligned}
& \|\mathbf{p}(t)-\boldsymbol{\pi}\| \leqslant \kappa e^{-\min _{i}\left\{\alpha_{i}\right\} t}\|\mathbf{p}(0)-\boldsymbol{\pi}\|, \quad t \geqslant 0, \\
& \|\mathbf{p}(t)-\boldsymbol{\pi}\| \geqslant \frac{1}{\kappa} e^{-\max _{i}\left\{\zeta_{i}\right\} t}\|\mathbf{p}(0)-\boldsymbol{\pi}\|, \quad t \geqslant 0,
\end{aligned}
$$

где $\kappa=4 \sum_{i=1}^{n}\left(d_{i} / d\right)$, а $\alpha_{i}$ и $\zeta_{i}$ определяются соответственно формулами (35) и (37). Кроме того, если $p_{i}(0) \geqslant \pi_{i}$ при $i=1,2, \ldots, n$ (или $p_{i}(0) \leqslant \pi_{i}$ nрu $\left.i=1,2, \ldots, n\right)$, mo

$$
\|\mathbf{p}(t)-\boldsymbol{\pi}\| \geqslant \frac{1}{\kappa} e^{-\max _{i}\left\{\alpha_{i}\right\} t}\|\mathbf{p}(0)-\boldsymbol{\pi}\|, \quad t \geqslant 0 .
$$

Д о к а з а т е л ь с т в о. Полагая $D=\operatorname{diag}\left(d_{1}, d_{2}, \ldots, d_{n}\right)$ и выбирая $T$ как в (32), получаем

$$
\|D T\|=\sum_{i=1}^{n} d_{i} \quad \text { и } \quad\left\|(D T)^{-1}\right\|=\frac{2}{d} .
$$

Оценки (38) и (40) вытекают теперь из теоремы 5 , а (39) следует из (18).

Отметим еще, что, в соответствии с теоремой 8 , наилучший показатель экспоненты в нижней оценке (39) равен $-\chi t$. В случае произвольного начального распределения (и $n>1$ ) этот показатель неулучшаем и несложно построить примеры, для которых $\|\mathbf{p}(t)-\boldsymbol{\pi}\|=O\left(e^{-\chi t}\right)$ при $t \rightarrow \infty$. Однако надо иметь в виду, что при больших значениях $t$ нижняя оценка (39) окажется (почти наверно) слишком грубой, поскольку спектральное представление для величин $p_{j}(t)-\pi_{j}$, а значит, и для $\|\mathbf{p}(t)-\boldsymbol{\pi}\|$, содержит и слагаемые порядка $e^{-\beta t}$, так что указанная общая оценка окажется точной при очень специальном выборе интенсивностей и начальных условий (если начальные условия, скажем, выбирать случайно, то с нулевой вероятностью).

В завершение этого пункта рассмотрим специальный пример ПРГ с постоянными (не зависящими от состояния) интенсивностями рождения $a_{i}=a, 0 \leqslant i<n$, и гибели $b_{i}=b, 0<i \leqslant n$. Чтобы отметить зависимость 
от размерности $n$, будем писать $\beta_{n}$ и $\chi_{n}$ вместо $\beta$ и $\chi$ соответственно. Выбирая

$$
d_{i}=\left(-\sqrt{\frac{b}{a}}\right)^{i-1} \frac{\sin (i n \pi /(n+1))}{\sin (n \pi /(n+1))}, \quad i=1, \ldots, n
$$

$\left(d_{i}\right.$ положительны, так как $\left.(i-1) \pi<i n \pi /(n+1)<i \pi\right)$, и используя тождество

$$
\sin \frac{(i+1) n \pi}{n+1}+\sin \frac{(i-1) n \pi}{n+1}=2 \sin \frac{i n \pi}{n+1} \cos \frac{n \pi}{n+1}, \quad i=1, \ldots, n,
$$

получаем, что величины $\alpha_{i}$ в $(35)$ и $\zeta_{i}$ в $(37)$ постоянны и вычисляются по формулам

$$
\alpha_{i}=a+b-2 \sqrt{a b} \cos \frac{\pi}{n+1}, \quad \zeta_{i}=a+b+2 \sqrt{a b} \cos \frac{\pi}{n+1},
$$

$i=1, \ldots, n$. А тогда в соответствии с теоремами 7 и 8 получаем

$$
\beta_{n}=a+b-2 \sqrt{a b} \cos \frac{\pi}{n+1}, \quad \chi_{n}=a+b+2 \sqrt{a b} \cos \frac{\pi}{n+1}, \quad n \geqslant 1 .
$$

Эти результаты хорошо известны (см., например $[26$, с. 13$])$ и $[12$, пример 2.3]), однако доказательство представляется новым. Из явных представлений вытекает, что

$$
\lim _{n \rightarrow \infty} \beta_{n}=(\sqrt{a}-\sqrt{b})^{2} \quad \text { и } \quad \lim _{n \rightarrow \infty} \chi_{n}=(\sqrt{a}+\sqrt{b})^{2},
$$

и получаем простейшие примеры асимптотических результатов, которые будут изучаться в следующих пунктах.

5. Модели среднего поля. В этом пункте мы рассмотрим спиновую систему $\Phi \equiv(\phi(t), t \geqslant 0)$ на полном графе с $n$ узлами (vertices, sites). Пространство состояний такого процесса есть множество всех $2^{n}$ бинарных векторов длины $n$, где $i$-я компонента вектора состояний принимает значение 0 , если $i$-е состояние свободно, и 1 - если оно занято. Процесс задается с помощью $2 n$ неотрицательных интенсивностей nepecкока (flip rates) $\lambda_{i}$ и $\mu_{i}, i=0,1, \ldots, n-1$, где $\lambda_{i}$ и $\mu_{i}$ - интенсивности рождения и гибели для свободного (или соответственно занятого) узла, имеющего $i$ занятых соседних узлов. Такие спиновые системы известны в статистической физике как модели среднего поля (mean-field models).

Обозначим $|\mathbf{a}|$ общее число единиц бинарного вектора а (так что $|\phi(t)|$ - общее число занятых узлов в момент $t)$ и положим $X(t):=|\phi(t)|$, $t \geqslant 0$; понятно, что $\mathscr{X}:=\{X(t), t \geqslant 0\}-$ процесс рождения и гибели с пространством состояний $\{0,1, \ldots, n\}$ и интенсивностями рождения и гибели $a_{i}=(n-i) \lambda_{i}, 0 \leqslant i<n$, и $b_{i}=i \mu_{i-1}, 0<i \leqslant n$, соответственно. Следовательно, скорость сходимости к стационарному режиму 
для модели среднего поля $\Phi$ характеризуется скоростью сходимости соответствующего ПРГ $\mathscr{X}$, а значит, для исследования сходимости моделей среднего поля можно применять методы предыдущего пункта. Этот подход был развит в работах [12], [13] и [15].

Так, например, с использованием теоремы 7 в [12] было показано, что если $\lambda_{i}+\mu_{i}=c$ при $0 \leqslant i \leqslant n-1$, то

$$
\beta_{n} \geqslant c-(n-1) \max _{i}\left\{\left|\lambda_{i}-\lambda_{i-1}\right|,\left|\mu_{i}-\mu_{i-1}\right|\right\}, \quad n \geqslant 1
$$

причем равенство достигается, если и только если $\lambda_{i}=\lambda_{0}+i h, \mu_{i}=$ $\mu_{0}-i h, 0 \leqslant i \leqslant n-1$, при некотором действительном $h$, в этом случае $\beta_{n}=c-(n-1) h, n \geqslant 1$. Аналогичным образом в [15] было показано, что при $\lambda_{i}+\mu_{i}=c$ значение $\chi_{n}$ находится точно, а именно, $\chi_{n}=n c, n \geqslant 1$. Как и в конце предыдущего пункта, зависимость от $n$ будем учитывать, записывая $\beta_{n}$ и $\chi_{n}$ вместо $\beta$ и $\chi$ соответственно.

Специальный класс моделей среднего поля с равномерным стационарным распределением возникает, если $\lambda_{i}=\mu_{i}, 0 \leqslant i \leqslant n-1$. Случай $\lambda_{i}=\mu_{i}=c(i+1)^{s}, 0 \leqslant i \leqslant n-1$, при некотором $c>0$ и $s \geqslant 0$ был рассмотрен в [13] и [15]. В частности, используя теорему 7, в [13] было показано, что

$$
\begin{aligned}
& \lim _{n \rightarrow \infty} \frac{\beta_{n}}{n^{s}}=2^{1-s} c, \quad 0 \leqslant s<1, \\
& \lim _{n \rightarrow \infty} \frac{\beta_{n}}{n}=c, \quad s \geqslant 1
\end{aligned}
$$

а асимптотика для $\chi_{n}$ была исследована в [15]. Формула (43) допускает обобщение, как показывает следующее утверждение.

Теорема 10. Пусть $\lambda_{0}=\mu_{0}=c>0$, кроме того, $\lambda_{i}=\mu_{i} \geqslant$ $c(i+1)$ при $0<i \leqslant n-1$. Тогда

$$
\lim _{n \rightarrow \infty} \frac{\beta_{n}}{n}=c .
$$

Д о к а 3 а т е л ь с т в о. Записывая $d_{1}=d / \lambda_{0}$ и выбирая $d_{i+1}:=$ $1 /\left(i \lambda_{i}\right)$ для $i=1,2, \ldots, n$, после небольших преобразований приходим к следующим формулам, определяющим $\alpha_{i}$ из (35):

$$
\alpha_{i}= \begin{cases}\lambda_{0}\left(\left(1-\frac{1}{d}\right) n+1+\frac{1}{d}\right), & i=1, \\ \frac{\lambda_{1}}{2}(n+4-2 d), & i=2, \\ \frac{\lambda_{i-1}}{i}\left(n-\frac{i}{i-2}\right), & i=3,4, \ldots, n .\end{cases}
$$


Теперь, выбирая $d:=\sqrt{n}$, получаем, что

$$
\frac{\beta_{n}}{n} \geqslant \min _{1 \leqslant i \leqslant n}\left\{\frac{\alpha_{i}}{n}\right\} \geqslant c(1+o(1)) \quad \text { при } \quad n \rightarrow \infty .
$$

С другой стороны, имеем

$$
\min _{1 \leqslant i \leqslant n} \alpha_{i} \leqslant \alpha_{1}=c\left(n+1-\frac{n-1}{d_{1}}\right) \leqslant c(n+o(n)) \quad \text { при } \quad n \rightarrow \infty
$$

для любого $d_{1}>0$, так что результат вытекает из минимаксного представления теоремы 7.

6. Система обслуживания $M / M / N / N+R$. В этом пункте мы рассмотрим систему обслуживания $M / M / N / N+R$, где $N \geqslant 1$ - число приборов (серверов), а $R \geqslant 0$ - число мест ожидания. Будем предполагать, что интенсивность поступления требований $\lambda(N)>0$ и число мест ожидания $R \equiv R(N)$ являются функциями от $N$. Если интенсивность обслуживания одним прибором есть $\mu$, то число требований в системе (длина очереди) есть ПРГ с интенсивностями

$$
a_{i}=\lambda(N), \quad 0 \leqslant i<n(N), \quad \text { и } \quad b_{i}=\min \{i, N\} \mu, \quad 0<i \leqslant n(N),
$$

где $n(N):=N+R(N)$. Исследуем здесь асимптотическое поведение при $N \rightarrow \infty$ параметра сходимости $\beta \equiv \beta_{n(N)}$, а также нижней границы скорости сходимости $\chi \equiv \chi_{n(N)}$. Первый результат относится к ситуации, когда $\lambda(N)$ есть константа.

Теорема 11. Пусть $\lambda(N)=\lambda>0$, тогда, независимо от поведения $R(N)$,

$$
\lim _{N \rightarrow \infty} \beta_{n(N)}=\mu .
$$

Д о к а з а т е л ь с т в о. Выбирая

$$
d_{i}= \begin{cases}1, & 1 \leqslant i \leqslant N, \\ \left(\frac{N}{N-1}\right)^{i-N}, & N<i<N+R(N), \\ \frac{(N-1) \mu}{(N-1) \mu+\lambda}\left(\frac{N}{N-1}\right)^{R(N)}, & i=N+R(N)(\text { если } R(N)>0),\end{cases}
$$

получаем после очевидных преобразований следующие равенства для величин $\alpha_{i}$ из (35):

$$
\alpha_{i}= \begin{cases}\mu, & 1 \leqslant i<N \quad \text { и } \quad i=N+R(N), \\ \mu-\frac{\lambda}{N-1}, & N \leqslant i<N+R(N)-1, \\ \mu-\frac{\lambda(\lambda-\mu)}{(N-1) \mu+\lambda}, & i=N+R(N)-1(\text { если } R(N)>1) .\end{cases}
$$


Следовательно, все $\alpha_{i}$ равны $\mu+o(1)$ при $N \rightarrow \infty$, а тогда по теореме 7 получаем требуемое утверждение.

Несложно убедиться, что в условиях теоремы 11 выполняется также и равенство

$$
\lim _{N \rightarrow \infty} \frac{\chi_{n(N)}}{N}=\mu \text {. }
$$

Действительно, выбирая $d_{i}=N^{i / 2}$ при $1 \leqslant i \leqslant n(N)$, получаем, что величины $\zeta_{i}$ из (37) удовлетворяют неравенствам $\zeta_{i} \leqslant N \mu+o(N)$, так что в соответствии с минимаксным представлением $(36)$ имеем $\chi_{n(N)} \leqslant N \mu+$ $o(N)$ при $N \rightarrow \infty$. С другой стороны, $\zeta_{N}>N \mu$ при любом выборе $d_{i}$, так что $\chi_{n(N)}>N \mu$.

Далее будет более подробно изучен специальный случай $R(N)=0$ (модель Эрланга, или система с потерями).

Как было показано в [1], в этой ситуации $\beta_{n(N)} \equiv \beta_{N}$ есть наименьший корень полинома

$$
S(x):=\frac{\lambda(N)}{x}\left\{c_{N+1}\left(\frac{x}{\mu}, \frac{\lambda(N)}{\mu}\right)-c_{N}\left(\frac{x}{\mu}, \frac{\lambda(N)}{\mu}\right)\right\},
$$

где

$$
c_{n}(x, a):=\sum_{m=0}^{n}(-1)^{m}\left(\begin{array}{c}
n \\
m
\end{array}\right)\left(\begin{array}{c}
x \\
m
\end{array}\right) \frac{m !}{a^{m}}, \quad n \geqslant 0,
$$

— полиномы Шарлье (см., например [5, раздел VI.1]). Поскольку полиномы Шарлье удовлетворяют рекуррентному соотношению

$$
a c_{n+1}(x, a)-a c_{n}(x, a)+x c_{n}(x-1, a)=0, \quad n \geqslant 0,
$$

можно записать

$$
S(x)=-c_{N}\left(\frac{x}{\mu}-1, \frac{\lambda(N)}{\mu}\right)
$$

а следовательно,

$$
\beta_{N}=\mu+\mu \xi_{N, 1}\left(\frac{\lambda(N)}{\mu}\right)
$$

где $\xi_{N, 1}(a)$ - минимальный корень полинома Шарлье $c_{N}(x, a)$. Известно, что $\xi_{N, 1}(a)>0$, так что

$$
\beta_{N}>\mu
$$

однако получение каких-либо явных выражений для $\xi_{N, 1}(a)$ в общем случае не представляется возможным. Тем не менее более подробную информацию об асимптотике $\beta_{N}$ при $N \rightarrow \infty$ получить можно. Случай постоянной $\lambda(N)$ рассмотрен в теореме 11, а теперь будет исследована более интересная ситуация, когда $\lambda(N)=\lambda N$, изучавшаяся, например, в работах [10] и [28].

В случае $\lambda=\mu$ можно найти точное значение $\beta_{N}$. Так, поскольку $c_{N}(x, a)=c_{x}(N, a)$ при натуральных $x$, имеем $c_{N}(1, N)=c_{1}(N, N)=0$. 
Однако полиномы Шарлье ортогональны по отношению к мере, состоящей из точечных масс в точках $0,1,2, \ldots$, так что из теории ортогональных полиномов (см. [5, гл. 2]) вытекает, что $i$-й наименьший корень полинома $c_{N}(x, a)$ больше, чем $i-1$, при $i=1,2, \ldots$ В качестве следствия отсюда получаем, что $\xi_{N, 1}(N)=1$, а значит, при всех $N \geqslant 1$

$$
\beta_{N}=2 \mu \quad \text { при } \quad \lambda=\mu .
$$

Другой способ доказательства того же равенства (с применением методов предшествующих пунктов) состоит в выборе $d_{k}=$ $(N-k)(N-k+1)^{-1}$, тогда получаем $\alpha_{k}=\lambda+\mu+k(N-k)^{-1}(\lambda-\mu)$, а значит, при $\lambda=\mu$ все $\alpha_{k}$ равны $\lambda+\mu=2 \mu$ и, следовательно, $\beta_{N}=2 \mu$.

Отметим также, что $c_{N}(1, a)=c_{1}(N, a)=1-N / a<0$ при $a<N$. С учетом того, что $c_{0}(0, a)=1$, отсюда вытекает оценка $\xi_{N, 1}(a)<1$ при $a<N$ и, значит,

$$
\beta_{N}<2 \mu, \text { если } \lambda<\mu \text {. }
$$

Используя нашу теорему 7, можно получить следующие асимптотические результаты, согласующиеся с (недоказанными) утверждениями из $[10]$.

Теорема 12. Пусть $\lambda(N)=\lambda N$, причем $\lambda>0$, тогда

$$
\begin{aligned}
& \lim _{N \rightarrow \infty} \beta_{N}=\mu, \quad \text { если } \quad \lambda<\mu, \\
& \lim _{N \rightarrow \infty} \frac{\beta_{N}}{N}=(\sqrt{\lambda}-\sqrt{\mu})^{2}, \quad \text { если } \quad \lambda>\mu .
\end{aligned}
$$

Д ок а з а те ль с т в о. Пусть вначале $\lambda<\mu$. Положим $L:=$ $[N \lambda / \mu]$ и выберем $d_{N+1}:=0, d_{N}:=(N-1) \mu$,

$$
d_{i}:=d_{i+1}+\left(d_{i+1}-d_{i+2}\right) \frac{N \lambda}{i \mu}, \quad i=N-1, N-2, \ldots, L,
$$

и $d_{i}:=d_{L}$ при $i<L$. Понятно, что $d_{i}>d_{i+1}$, если $i=L, L+1, \ldots, N$, а значит, $d_{i}>0$ при $1 \leqslant i \leqslant N$. Подставляя эти $d_{i}$ в $(35)$, получаем, что $\alpha_{i}=\mu$ при $i \neq L$. Далее,

$$
0<1-\frac{d_{i+1}}{d_{i}}=\frac{d_{i+1}}{d_{i}}\left(1-\frac{d_{i+2}}{d_{i+1}}\right) \frac{N \lambda}{i \mu}<\left(1-\frac{d_{i+2}}{d_{i+1}}\right) \frac{N \lambda}{i \mu}, \quad L \leqslant i<N,
$$

так что

$$
1-\frac{d_{L+1}}{d_{L}}<\frac{N \lambda}{L \mu} \cdots \frac{N \lambda}{(N-1) \mu}<\left(\frac{\lambda}{\mu}\right)^{N-L} .
$$

В результате получаем

$$
\alpha_{L}=\mu+\left(1-\frac{d_{L+1}}{d_{L}}\right) N \lambda<\mu+N \lambda\left(\frac{\lambda}{\mu}\right)^{N-L}=\mu+o(1) \quad \text { при } \quad N \rightarrow \infty,
$$


что, с учетом (47) и минимаксного представления теоремы 7 , дает первое утверждение теоремы.

Пусть теперь $\lambda>\mu$. Выбирая $d_{i}:=(\mu / \lambda)^{i / 2}, i=1,2, \ldots, N$, получаем величины $\alpha_{i}$ из $(35)$ в виде

$$
\alpha_{i}= \begin{cases}(\lambda-\sqrt{\lambda \mu}) N-(\sqrt{\lambda \mu}-\mu) i+\sqrt{\lambda \mu}, & 1 \leqslant i<N, \\ \lambda N-(\sqrt{\lambda \mu}-\mu) N+\sqrt{\lambda \mu}, & i=N,\end{cases}
$$

так что $\alpha_{N-1}=\min _{i}\left\{\alpha_{i}\right\}$. По теореме 7 имеем теперь

$$
\beta_{N} \geqslant \alpha_{N-1}=(\sqrt{\lambda}-\sqrt{\mu})^{2} N+2 \sqrt{\lambda \mu}-\mu .
$$

С другой стороны, полагая $M_{1}:=[N-\sqrt{N}]$ и $M_{2}:=\left[N-\frac{1}{2} \sqrt{N}\right]$ и выбирая

И

$$
d_{i}:=\left(2 \sqrt{\frac{\mu}{\lambda}}\right)^{i}, \quad 1 \leqslant i<M_{1},
$$

$$
d_{i+1}:= \begin{cases}\frac{i-M_{1}+3}{i-M_{1}+2} \sqrt{\frac{\mu}{\lambda}} d_{i}, & M_{1} \leqslant i<M_{2}, \\ \sqrt{\frac{\mu}{\lambda}} d_{i}, & i=M_{2}, \\ \frac{N-i}{N-i+1} \sqrt{\frac{\mu}{\lambda}} d_{i}, & M_{2}<i<N,\end{cases}
$$

получаем после очевидных преобразований оценки

$$
\alpha_{i} \leqslant(\sqrt{\lambda}-\sqrt{\mu})^{2}+o(N) \quad \text { при } \quad N \rightarrow \infty, \quad 1 \leqslant i \leqslant N .
$$

А тогда, в соответствии с минимаксным представлением теоремы 7 , получаем

$$
\beta_{N} \leqslant(\sqrt{\lambda}-\sqrt{\mu})^{2} N+o(N) \quad \text { при } \quad N \rightarrow \infty,
$$

что вместе с (50) дает второе утверждение теоремы.

3 а м е ч а н и е 1 . Используя совершенно иную технику, в [19, теорема 3] показано, что $\xi_{N, 1}(a N)>(\sqrt{a}-1)^{2} N+c \sqrt[3]{N}$ при $a>1$ для некоторых положительных постоянных $c$, так что (50) может быть заменено на $\beta_{N}>(\sqrt{\lambda}-\sqrt{\mu})^{2} N+c \mu \sqrt[3]{N}$.

3 а м е ч а н и е 2. Другое доказательство (53) можно получить, используя схему рассуждений леммы 36 из [21, гл. 17] и соотношение

$$
a c_{n}(x+1, a)+(n-a-x) c_{n}(x, a)+x c_{n}(x-1, a)=0, \quad n \geqslant 0 .
$$

(Этот подход был предложен И. Красиковым.)

Можно использовать $\mathbf{d}$ из доказательства предыдущей теоремы, чтобы получить верхние оценки для $\|\mathbf{p}(t)-\boldsymbol{\pi}\|$ с помощью оценки (38). А именно, получаем:

$$
\|\mathbf{p}(t)-\boldsymbol{\pi}\| \leqslant\left\{\begin{array}{ll}
C_{1} e^{-\left\{N(\sqrt{\lambda}-\sqrt{\mu})^{2}+2 \sqrt{\lambda \mu}-\mu\right\} t} & \text { при } \lambda>\mu, \\
C_{2} e^{-\mu t} & \text { при } \lambda<\mu,
\end{array} \quad t \geqslant 0,\right.
$$


где постоянные $C_{1}$ и $C_{2}$ зависят от $N$. Эти оценки согласуются с неравенствами из [10, утверждения 6 и 10].

Рассмотрим, наконец, асимптотическое поведение $\chi_{N}$ при $N \rightarrow \infty$ в условиях предыдущей теоремы.

Теорема 13. Пусть $\lambda(N)=\lambda N$ при $\lambda>0$, тогда

$$
\lim _{N \rightarrow \infty} \frac{\chi_{N}}{N}=(\sqrt{\lambda}+\sqrt{\mu})^{2} .
$$

Д о к а з а т е л ь с т в о. Выбирая $d_{i}:=(\mu / \lambda)^{i / 2}, i=1, \ldots, N$, получаем величины $\zeta_{i}$ из (37) в виде:

$$
\zeta_{i}= \begin{cases}(\lambda+\sqrt{\lambda \mu}) N+(\sqrt{\lambda \mu}+\mu) i-\sqrt{\lambda \mu}, & 1 \leqslant i<N, \\ \lambda N+(\sqrt{\lambda \mu}+\mu) N-\sqrt{\lambda \mu}, & i=N,\end{cases}
$$

так что при достаточно больших $N$ имеем $\zeta_{N-1}=\max _{i}\left\{\zeta_{i}\right\}$. Тогда из (36) следует, что

$$
\chi_{N} \leqslant \zeta_{N-1}=(\sqrt{\lambda}+\sqrt{\mu})^{2} N-2 \sqrt{\lambda \mu}-\mu .
$$

С другой стороны, выбирая $d_{i}$ так же, как в (51) и (52), получаем оценки

$$
\zeta_{i} \geqslant(\sqrt{\lambda}+\sqrt{\mu})^{2}+o(N) \quad \text { при } \quad N \rightarrow \infty, \quad 1 \leqslant i \leqslant N,
$$

а значит, с учетом минимаксного представления (36), имеем

$$
\chi_{N} \geqslant(\sqrt{\lambda}+\sqrt{\mu})^{2} N+o(N) \text { при } \quad N \rightarrow \infty .
$$

Наконец, применяя (55) и (56), получаем утверждение теоремы.

Авторы благодарны Илье Красикову за полезные обсуждения.

\section{СПИСОК ЛИТЕРАТУРЫ}

1. Blanc J. P. C., van Doorn E. A. Relaxation times for queueing systems. - Mathematics and Computer Science (Amsterdam, 1983). Ed. by J. W. de Bakker, M. Hazewinkel, and J. K. Lenstra. Amsterdam: North-Holland, 1986, p. 139-162. (CWI Monogr., v. 1.)

2. Chen M.-F. Estimation of spectral gap for Markov chains. - Acta Math. Sinica (N.S.), 1996, v. 12, № 4, p. 337-360.

3. Chen M.-F. Variational formulas and approximation theorems for the first eigenvalue in dimension one. - Sci. China Ser. A, 2001, v. 44, № 4, p. 409-418.

4. Chen M.-F. Eigenvalues, Inequalities, and Ergodic Theory. London: Springer-Verlag, 2005, 228 p.

5. Chihara T.S. An Introduction to Orthogonal Polynomials. New York: Gordon and Breach, 1978, 249 p.

6. Dahlquist G. Stability and Error Bounds in the Numerical Integration of Ordinary Differential Equations. Inaugural dissertation (University of Stockholm). Uppsala: Almqvist \& Wiksells Boktryckeri AB, 1958, 87 p. Reprinted in: Transactions of the Royal Institute of Technology, № 130. Stockholm, 1959. 
7. van Doorn E. A. Conditions for exponential ergodicity and bounds for the decay parameter of a birth-death process. - Adv. Appl. Probab., 1985, v. 17, № 3, p. 514-530.

8. van Doorn E. A. Representations for the rate of convergence of birth-death processes. - Theory Probab. Math. Statist., 2002, v. 65, p. 37-43.

9. van Doorn E.A., van Foreest N.D., Zeifman A.I. Representations for the extreme zeros of orthogonal polynomials. - J. Comput. Appl. Math. (to appear); http://eprints.eemcs.utwente.nl/10994/

10. Fricker C., Robert P., Tibi D. On the rates of convergence of Erlang's model. - J. Appl. Probab., 1999, v. 36, № 4, p. 1167-1184.

11. Гнеденко Б. В., Макаров И. П. Свойства решений задачи с потерями в случае периодических интенсивностей. - Дифференц. уравнения, 1971, т. 7, с. 1696-1698.

12. Granovsky B.L., Zeifman A.I. The decay function of nonhomogeneous birth-death processes, with application to mean-field models. - Stochastic Process. Appl., 1997, v. 72 , № 1 , p. $105-120$.

13. Granovsky B.L., Zeifman A.I. The $N$-limit of spectral gap of a class of birth-death Markov chains. - Appl. Stoch. Models Bus. Ind., 2000, v. 16, № 4, p. 235-248.

14. Granovsky B. L., Zeifman A.I. Nonstationary queues: estimation of the rate of convergence. - Queueing Syst., 2004, v. 46, № 3, p. 363-388.

15. Грановский Б. Л., Зейфман А.И. О нижней границе спектра для некоторых моделей среднего поля. - Теория вероятн. и ее примен., 2004, т. 49, в. 1, с. 164-171.

16. Karlin S., McGregor J. L. The differential equations of birth-and-death processes, and the Stieltjes moment problem. - Trans. Amer. Math. Soc., 1957, v. 85, p. 489-546.

17. Karlin S., McGregor J.L. Ehrenfest urn models. - J. Appl. Probab., 1965, v. 2, p. $352-376$.

18. Kijima $M$. Evaluation of the decay parameter for some specialized birth-death processes. - J. Appl. Probab., 1992, v. 29, № 4, p. 781-791.

19. Krasikov I. Bounds for zeros of the Charlier polynomials. - Methods Appl. Anal., 2002, v. 9, № 4, p. 599-610.

20. Лозинский C.M. Оценка погрешности численного интегрирования обыкновенного дифференциального уравнения, І. - Изв. вузов. Матем., 1958, в. 5, с. 52-90; исправл.: 1959 , в. 5 , с. 222.

21. MacWilliams F. J., Sloane N. J. A. The Theory of Error-Correcting Codes. Part II. Amsterdam: North-Holland, 1977, 392 p.

22. Meyer C. D. Matrix Analysis and Applied Linear Algebra. Philadelphia: SIAM, 2001, 718 p. (Updates available on http://www.matrixanalysis.com).

23. Рудин У. Функциональный анализ. М.: Мир, 1975, 443 с.

24. Söderlind $G$. The logarithmic norm. History and modern theory. - BIT. Numerical Mathematics, 2006, v. 46, № 3, p. 631-652.

25. Ström T. On logarithmic norms. - SIAM J. Numer. Anal., 1975, v. 12, № 5, p. 741753.

26. Takács L. Introduction to the Theory of Queues. New York: Oxford Univ. Press, 1962, $268 \mathrm{p}$

27. Varga R. S. Matrix Iterative Analysis. Berlin: Springer-Verlag, 2000, 358 p.

28. Voit $M$. A note on the rate of convergence to equilibrium for Erlang's model in the subcritical case. - J. Appl. Probab., 2000, v. 37, № 3, p. 918-923.

29. Zeifman A.I. Some estimates of the rate of convergence for birth and death processes. - J. Appl. Probab., 1991, v. 28, № 2, p. 268-277.

30. Zeifman A.I. On the estimation of probabilities for birth and death processes. - J. Appl. Probab., 1995, v. 32, № 3, p. 623-634.

31. Zeifman A.I. Upper and lower bounds on the rate of convergence for nonhomogeneous birth and death processes. - Stochastic Process. Appl., 1995, v. 59, № 1, p. 157-173.

32. Зейфман А.И., Бенинг В. Е., Соколов И. А. Марковские цепи и модели с непрерывным временем. М.: ЭЛЕКС-КМ, 2008. 